\title{
Erector spinae plane block versus thoracic epidural analgesia in video-assisted thoracic surgery: a study protocol for a prospective randomized open label non-inferiority trial
}

Renee van den Broek ( $\square$ renee.vd.broek@catharinaziekenhuis.nl )

Catharina Ziekenhuis https://orcid.org/0000-0001-5939-695X

\section{Seppe Koopman}

Maasstad Ziekenhuis

Jonne Postema

Maasstad Ziekenhuis

Niels Verberkmoes

Catharina Ziekenhuis

Ki Jinn Chin

University of Toronto

Arthur Bouwman

Catharina Ziekenhuis

Barbara Versyck

AZ Turnhout

Study protocol

Keywords: Erector spinae plane block, thoracic epidural analgesia, regional anesthesia, postoperative pain, video-assisted thoracoscopic surgery, pain management.

Posted Date: February 2nd, 2021

DOI: https://doi.org/10.21203/rs.3.rs-22893/v1

License: (c) (1) This work is licensed under a Creative Commons Attribution 4.0 International License. Read Full License 


\section{Abstract}

Background: Thoracic epidural analgesia is considered the gold standard for pain relief in video-assisted thoracoscopic surgery. This neuraxial technique blocks pain sensation by injecting local anesthetic in the epidural space near the spinal cord to block spinal nerve roots. Recently, the erector spinae plane block has been introduced as a practical alternative to the thoracic epidural. This interfascial regional anesthesia technique interrupts pain sensation by injecting local anesthetic in between the muscular layers of the thoracic wall. Several case series and three RCTs described it as an effective pain management technique in video-assisted thoracoscopic surgery. (1-5)

The objective of this study is to test the hypothesis that a continuous erector spinae plane block is noninferior in terms of the quality of recovery as measured by the Quality of Recovery-15 score compared to continuous thoracic epidural analgesia in patients undergoing elective unilateral video-assisted thoracoscopic surgery.

Methods: This is a prospective randomized open label non-inferiority trial. A total of 90 adult patients undergoing video-assisted thoracoscopic surgery will be randomized 1:1 to receive either pain treatment with continuous erector spinae plane block (study group) or continuous thoracic epidural analgesia (control group). The primary endpoint is the quality of recovery as measured by the Quality of Recovery15 score. Secondary endpoints are postoperative pain as Numerical Rating Score scores, length of hospital stay, failure of analgesic technique, postoperative morphine-equivalent consumption, itching, nausea and vomiting, total operative time, complications related to surgery, perioperative hypotension, complications related to pain treatment, duration of bladder catheterization, time of first assisted mobilization $>20$ meters and of mobilization to sitting in a chair.

Discussion: This randomized controlled trial aims to confirm whether a continuous erector spinae plane block can equal analgesic effect as regional anesthesia technique compared with a thoracic epidural in patients undergoing video-assisted thoracoscopic surgery.

\section{Administrative Information}

Note: the numbers in curly brackets in this protocol refer to SPIRIT checklist item numbers. The order of the items has been modified to group similar items (see http://www.equator-network.org/reporting-guidelines/spirit2013-statement-defining-standard-protocol-items-for-clinical-trials/). 


\begin{tabular}{|c|c|}
\hline Title $\{1\}$ & $\begin{array}{l}\text { rector spinae plane block versus thoracic epidural analgesia in video-assisted thoracic } \\
\text { argery: a study protocol for a prospective randomized open label non-inferiority trial }\end{array}$ \\
\hline $\begin{array}{l}\text { Trial } \\
\text { registration } \\
\{2 \mathrm{a} \quad \text { and } \\
2 \mathrm{~b}\} .\end{array}$ & 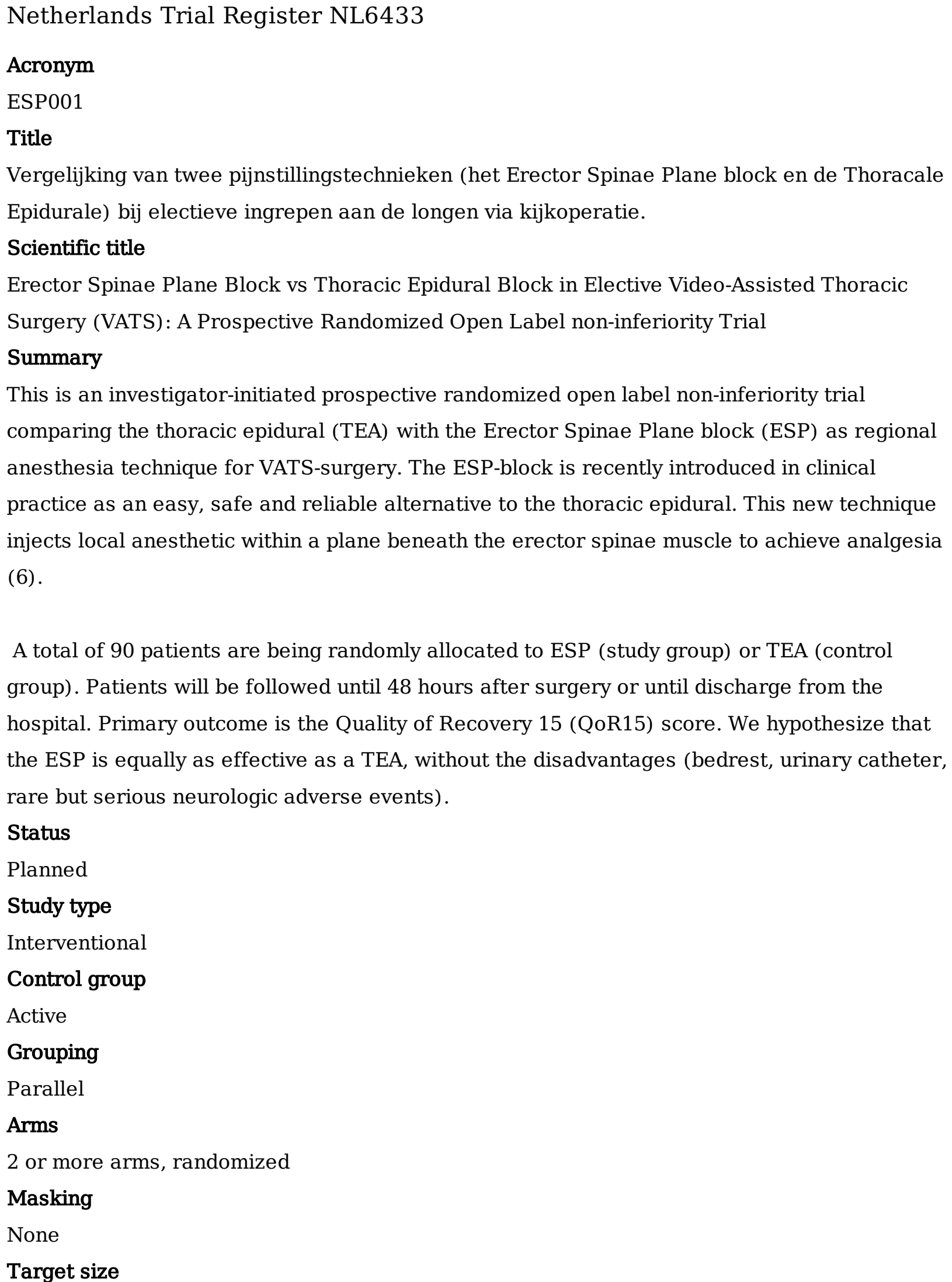 \\
\hline
\end{tabular}




\section{Inclusion criteria}

In order to be eligible to participate in this study, a subject must meet all of the following criteria: (1) Age between 18 and 75 years old, (2) BMI between 20 and 30kg/m 2 , (3) scheduled for elective VATS, and (4) written informed consent.

\section{Exclusion criteria}

Exclusion criteria are as follows: (1) ASA status 4, (2) chronic opioid use ( $>3$ months of strong opioids, weak opioids such as tramadol are allowed), (3) renal or liver failure inhibiting the systematic use of paracetamol and/or NSAIDs, (4) contraindication for epidural analgesia (e.g. INR or platelets according to local protocol, local infection at the surgery site or puncture site) (5) allergy to study medication, (6) pregnancy, (7) cognitive impairment, (8) insufficient comprehension of the Dutch QoR-40 questionnaire.

\section{Start date}

2019-01-01

\section{Stop date}

2020-01-01

\section{Diseases}

In English: Video Assisted Thoracic Surgery (VATS) Thoracic Epidural Analgesia (TEA) Erector Spinae Plane block In het Nederlands Video Geassisteerde Thoracale Chirurgie Thoracale Epidurale Erector Spinae Plane block

\section{Hypothesis}

Effective postoperative pain control is an essential and humanitarian need of every surgical procedure. Inadequate pain control may result in increased mortality, delayed recovery and increased hospital costs (1). The optimal perioperative analgesic strategy is preemptive and involves the combined administration of local anesthetic techniques [local anesthetic infiltration, peripheral nerve blocks, and neuraxial blocks (epidural and paravertebral)], systemic analgesic agents (opioids, acetaminophen, non-steroidal anti-inflammatory drugs, and cyclooxygenase-2-specific inhibitors) and analgesic adjuncts such as steroids, ketamine, $\alpha-2$ agonists, and anticonvulsants (2). This so-called multimodal approach improves the analgesic effect because of the synergizing effect between the different analgesia techniques and/or drugs.

Up until now, the epidural analgesia is the gold standard local anesthetic technique for VATS surgery (3). However, the invasiveness of this technique, the rare but serious neurologic complications and the failure rates up to $30 \%$ (4) have resulted in a search for alternatives. Alternatives include lower thoracic catheter placement, intercostal nerve blocks, paravertebral blocks, intrapleural catheters, local anesthetic infiltration, and systemic analgesia with one or more agents (4). However, none of these techniques were able to replace the thoracic epidural as gold standard due to (5) 'too technically challenging' or 'insufficient analgesia' (6). Recently the ESP-block has been introduced in clinical practice as 
an easy, safe and reliable alternative to the thoracic epidural. This new technique injects local anesthetic within a plane beneath the erector spinae muscle to achieve analgesia (6). The most significant advantage of the ESP-block is its simplicity and safety. The sonoanatomy is easily recognizable and there are no structures at risk of needle injury in the immediate vicinity (6). Case reports describe the successful application of the ESP-block for analgesia after VATS surgery $(7,8)$, but evidence from large trials targeting a specific surgical population is lacking. To test the hypothesis that the ESP block with Continuous ESP Analgesia (ESP) is non-inferior in terms of the quality of recovery as measured by the Quality of Recovery 15 (QoR15) score compared to the thoracic epidural with continuous epidural analgesia (TEA) for patients undergoing elective unilateral VATS.

\section{Interventions}

Patients will be randomized (1:1) to receive either pain treatment with ESP (the study group) or TEA (the control group). Patients in the intervention group receive continuous ESP analgesia (ESP) with bupivacaine $0.125 \%$ and a PCIA pump with morphine. Settings of the continuous ESP analgesia are $10-15 \mathrm{ml} / \mathrm{h}$, settings of the PCIA pump are according to local protocol. When ESP does not provide adequate pain relief, minor adjustments or a manual topup are allowed. Patients in the control group receive thoracic epidural analgesia (TEA) through a continuous epidural analgesia (CEA) pump with bupivacaine $0.125 \%+$ sufentanil $1 \mathrm{mcg} / \mathrm{ml}$ at $8-12 \mathrm{ml} / \mathrm{h}$. When CEA does not provide adequate pain relief, minor adjustments or a manual top-up are allowed.

\section{Primary outcome}

The primary outcome of this study is the QoR15 (See section 6.1.) on POD1 and 2 including subscale analysis Comfort; Emotions, Independence, Support and Pain).

\section{Secondary outcome}

Secondary endpoints include

- postoperative Visual

Analog Scales (VAS) score on day 1 and 2 (assessed at rest and when moving (coughing) in the morning and in the evening)

- Length of hospital stay (LOS),

- requirement of rescue medication

- postoperative morphine-equivalent consumption per day,

- failure of analgesic technique (defined as catheter

failure, need for specialist intervention and/ or need for

rescue medication) (Table 1 )

- the total operative time (recorded as total time spent in the operating room, anesthetic time, surgical time), - complications related to surgery (e.g. bleeding, surgical 
site infection, conversion to open procedure),

- complications related to pain treatment (e.g. epidural

hematoma and abscess or local anesthetic toxicity)

- duration of bladder catheterization,

- first mobilization to chair and $>20$ meters

- 30 days post-surgical evaluation by phone of pain,

opioid use and patient satisfaction

Sponsors

Investigator initiated study.

Time points

Patients will be followed until 48 hours after surgery or until discharge from the hospital.

Thirty days after the surgery, patients will be contacted for a telephone survey.

\section{MEC approved}

No

Multicenter

Yes

\section{Randomised}

Yes

Plan to share IPD

N/A

IPD plan description

N/A

https://www.trialregister.nl/trial/6433

Protocol

Protocol version 5; 22/11/2019.

version $\{3\}$

Funding $\{4\}$

This work was supported by the Stichting Onderzoeksfonds Catharina Ziekenhuis (Foundation Research Fund Catharina Hospital) (Project 2018-7). It will cover the cost for a research nurse who will enter the data in the database and publication costs.

Author

details $\{5 \mathrm{a}\}$

R.J.C. (Renee) van den Broek MD (corresponding author)

Department of Anesthesiology and Pain medicine, Catharina hospital

Michelangelolaan 2, 5623 EJ Eindhoven, The Netherlands

Renee.vd.broek@catharinaziekenhuis.nl

Activities: drafted the manuscript and is the main researcher of the study at Catharina hospital

J.S.H.A. (Seppe) Koopman MD, PhD, MSc

Department of Anesthesiology and Pain Medicine, Maasstad Hospital

Maasstadweg 21, 3079 DZ, Rotterdam, the Netherlands

KoopmanJ@maasstadziekenhuis.nl 
Activities: revised the manuscript and is the main researcher in the Maasstad Hospital

J.M.C. (Jonne) Postema MD

Department of Anesthesiology and Pain Medicine, Maasstad Hospital

Maasstadweg 21, 3079 DZ, Rotterdam, the Netherlands

PostemaJ@maasstadziekenuis.nl

Activities: revised the manuscript and will conduct the study in the Maasstad Hospital

N.J. (Niels) Verberkmoes MD

Heart Center Catharina Hospital

Michelangelolaan 2, 5623 EJ Eindhoven, the Netherlands

Niels.verberkmoes@catharinaziekenhuis.nl

Activities: revised the manuscript

Ki Jinn Chin MBBS, FRCPC

Department of Anesthesia, Toronto Western Hospital,

339 Bathurst st, M5T 2S8 Toronto, Ontario, Canada

gasgenie@gmail.com

Activities: helped design the study, revised the manuscript

R.A. (Arthur) Bouwman MD, PhD

Department of Anesthesiology and Pain Medicine, Catharina hospital

Michelangelolaan 2, 5623 EJ Eindhoven, The Netherlands

Arthur.bouwman@catharinaziekenhuis.nl

Activities: helped design the study, contributed to and revised the manuscript

\section{B.J.B (Barbara) Versyck MD}

Department of anesthesiology and pain medicine, Catharina hospital

Michelangelolaan 2, 5623 EJ Eindhoven, The Netherlands

Department of Anesthesiology and Pain Medicine, AZ Turnhout

Steenweg op Merksplas 44, 2300 Turnhout, België

Barbara.versyck@azturnhout.be

Activities: conceived the project, obtained funding for the project, designed the study,

contributed to and revised the manuscript

Name and

contact

information

for the trial

sponsor

$\{5 b\}$

Role of

sponsor

$\{5 c\}$
Catharina Hospital, board of directors

Department research and education, Catharina hospital

Michelangelolaan 2, 5623 EJ Eindhoven, The Netherlands

+31402396520

This funding source had no role in the design of this study and will not have any role during its execution, analyses, interpretation of the data, or decision to submit results

The sponsor has no role in the design, execution, analysis, interpretation of the study or the decision to submit results. The role of the sponsor is according to GCP guidelines. 


\section{Introduction}

\section{Background and rationale $\{6 a\}$}

Thoracic epidural analgesia (TEA) is considered the gold standard analgesic technique for video-assisted thoracoscopic surgery (VATS).(6) The invasiveness of this technique, the rare but serious neurologic complications and the failure rates up to $30 \%$ (7), show the shortcomings of epidural analgesia. Also, neuraxial techniques are contra-indicated in patients using anticoagulation apart from acetylsalicylic acid and other non-steroidal anti-inflammatory drugs (NSAIDs). (8) Sepsis and infection are relative contraindications. (9) This has fuelled the interest in alternatives to TEA. Alternatives include intercostal nerve blocks, paravertebral blocks, intrapleural catheters, local anesthetic infiltration, and systemic analgesia with one or more agents. (6) However, none of these techniques were able to replace the thoracic epidural as gold standard due to being 'too technically challenging' (10) or providing 'insufficient analgesia'. (11)

The most recently-described alternative to TEA is the erector spinae plane (ESP) block. The ESP block is a fascial plane block that aims to inject local anesthetic within a plane beneath the erector spinae muscle. (11) The most significant advantages of the ESP block are its perceived simplicity and safety. The sonoanatomy is easily recognizable and there are no structures at risk of needle injury in the immediate vicinity. (11) The use of anticoagulation is not an absolute contra-indication to this technique. $(12,13)$ Multiple case reports have described its successful application for analgesia after VATS, $(1,2)$ and two RCTs reported that a single-injection ESP block lowers pain scores after VATS compared to no intervention. $(4,5)$ Two other RCTs have found continuous ESP block to be non-inferior to paravertebral block and TEA for analgesia in VATS during the first 24 hours postoperatively. $(3,14)$ However further evidence from multicenter trials comparing continuous ESP block to TEA in VATS is required to confirm these preliminary findings. We hypothesize that continuous ESP is non-inferior to continuous TEA, without the mentioned disadvantages. In this trial, we aim to compare the effect of ESP versus TEA on overall postoperative recovery after VATS.

\section{Objectives \{7\}}

Our primary objective is to test the hypothesis that continuous ESP is non-inferior to continuous TEA in terms of the quality of recovery as measured by the Quality of Recovery-15 (QoR-15) score in patients undergoing elective unilateral VATS on postoperative day (POD) 1 and 2. (15)

\section{Trial design $\{8\}$}

This is an investigator-initiated prospective randomized open label non-inferiority trial. It is designed as a non-inferiority trial as the analgesic effectiveness of a successful TEA is unquestioned. Furthermore, it is not necessary to demonstrate the analgesic superiority of the ESP block because of the previouslymentioned advantages it has over TEA. A double-blinded study protocol is not possible as the differences 
between continuous ESP block and TEA will be readily apparent during the study period; hence we have adopted an open-label study design.

\section{Methods: Participants, Interventions And Outcomes}

\section{Study setting $\{9\}$}

The study will be performed in the Catharina Hospital, Eindhoven, the Netherlands and Maasstad Hospital, Rotterdam, the Netherlands. Both centers are large teaching hospitals. The trial was approved by the Medical Research Ethics Committees United (MEC-U), Nieuwegein, the Netherlands. Clinical Trial Registration: Netherlands Trial Register (NL6433). All study participants provided written informed consent before randomization.

\section{Eligibility criteria $\{10\}$}

All adult patients scheduled for elective VATS (complete VATS, as described by Swanson and Shigemura) $(16,17)$ for lobectomy or wedge resection under general anesthesia in the Catharina Hospital Eindhoven or Maasstad Hospital Rotterdam will be asked for informed consent during the preoperative anesthesiology consultation. We plan to start recruitment in July 2020.

In order to be eligible to participate in this study, a subject must meet all of the following criteria: (1) age between 18 and 75 years old, (2) BMI between 18 and $30 \mathrm{~kg} / \mathrm{m} 2$, (3) scheduled for elective VATS, and (4) written informed consent.

Exclusion criteria are as follows: (1) ASA status 4 or 5, (2) chronic opioid use, defined as $>3$ months of opioid use (excluding tramadol and codeine), (3) renal or liver failure inhibiting the systematic use of paracetamol and/or NSAIDs, (4) contraindications to epidural analgesia including abnormal coagulation status, local infection, pre-existing neurological deficits of the torso or lower limbs, and spinal disease (5) allergy to study medication, (6) pregnancy, (7) cognitive impairment, (8) insufficient comprehension of the Dutch QoR-15 questionnaire.

\section{Who will take informed consent? $\{26 \mathrm{a}\}$}

Informed consent will be obtained by an anesthesiologist during the preoperative anesthesiology consultation. An informed consent form will be signed by both the patient and the anesthesiologist.

Additional consent provisions for collection and use of participant data and biological specimens $\{26 \mathrm{~b}\}$

N/A, no biological specimens are collected.

\section{Interventions}

Explanation for the choice of comparators $\{6 \mathrm{~b}\}$ 
The control group will receive thoracic epidural analgesia (TEA). This is considered the gold standard analgesic technique for video-assisted thoracoscopic surgery (VATS). (6)

\section{Intervention description $\{11 \mathrm{a}\}$}

Investigational treatment

During the study, patients will receive standard preoperative care. Both techniques will be performed after placement of an intravenous (IV) line and application of standard vital sign monitors (non-invasive blood pressure, electrocardiogram, and oxygen saturation). The TEA and the ESP block will be performed before the start of surgery, according to institutional protocol. The ESP block will be performed under ultrasound guidance and the thoracic epidural will be performed using the conventional landmark-guided technique in line with currently-accepted practice. All interventions will be performed consultant anesthesiologists experienced in the technique.

\section{Intervention group: Continuous ESP block}

The ESP block will be placed as described by Forero et al. (11) The patient will be installed in the lateral or sitting position. An ultrasound probe will be placed in a longitudinal position 2-3 cm lateral of the vertebral column. The erector spinae muscles will be identified in relation to the ipsilateral fifth thoracic vertebra (T5) transverse process. A Tuohy needle will be inserted with an in-plane technique in a caudal to cephalad direction until bony contact with the transverse process is obtained. Hydrodissection with normal saline will be performed to identify and open up the correct plane for injection. A loading dose of ropivacaine will be injected followed by the insertion of an 18-gauge catheter $5 \mathrm{~cm}$ beyond the needle tip. Patients over $70 \mathrm{~kg}$ will receive $200 \mathrm{mg}$ ropivacaine $(40 \mathrm{ml})$, patients $50-70 \mathrm{~kg}$ will receive $150 \mathrm{mg}$ ropivacaine $(40 \mathrm{ml})$ and patients under $50 \mathrm{~kg}$ will receive ropivacaine $3 \mathrm{mg} / \mathrm{kg}(40 \mathrm{ml})$. At the end of surgery, ESP catheter is used at a continuous rate of $5 \mathrm{ml} / \mathrm{h}$ of bupivacaine $0.125 \%$ and an extra bolus of $10 \mathrm{ml}$ bupivacaine $0.125 \%$ is given. Current institutional practice with other regional anesthesia techniques is to administer either ropivacaine at the start of surgery or bupivacaine at the end and continuous infusion on the ward. Because this techniques needs both a bolus at the start and end/ continuous infusion on the ward, we choose to adhere to known local practice as much as possible and use two different local anesthetics.

\section{Control group: Continuous thoracic epidural analgesia}

The epidural catheter will be inserted preoperatively at the T5-T7 vertebral level; the exact level will be at the discretion of the attending anesthesiologist. A loading dose of bupivacaine $0.25 \%$ ( $\max 10 \mathrm{ml}$ ) or ropivacaine $0.75 \%$ ( $\max 10 \mathrm{ml}$ ) will be administered, followed by an intraoperative infusion of 8-12 ml/h of bupivacaine $0.125 \%$ with sufentanil $1 \mu \mathrm{g} / \mathrm{ml}$ or $5 \mathrm{ml} /$ hour ropivacaine $0.2 \%$ with sufentanil $0.5 \mu \mathrm{g} / \mathrm{ml}$. These dosing regimens are in line with existing individual institutional protocols; the specific details will be left to the discretion of the attending anesthesiologist. 
Postoperatively we will not measure blocked sensory dermatomes in both ESP and TEA group as part of standard treatment. For TEA it is our current practice to measure blocked sensory dermatomes only in patients who have a NRS >4. For ESP block patients, other studies showed highly variable sensory blockade. $(18,19)$ Therefore, we do not measure it.

\section{General treatment regimen}

Induction of anesthesia, intraoperative hemodynamic management, and mechanical ventilation will follow current standards of care for both groups. In the operating room, general anesthesia is induced with propofol, sufentanil for analgesia and rocuronium for paralysis. The trachea is intubated, and the lungs are mechanically ventilated with pressure-regulated volume-controlled ventilation. After induction, general anesthesia is maintained with a propofol infusion and supplemented as needed by additional boluses of sufentanil for intraoperative analgesia. A radial arterial line and an internal jugular central venous line will be inserted at the discretion of the attending anesthesiologist. A urinary catheter is inserted for the patients in the TEA group but only if indicated in patients in the ESP group. All patients without urinary catheter will receive a bladder scan in the post-anesthetic care unit (PACU) before discharge to the ward as part of standard postoperative care. Cefuroxime $1500 \mathrm{mg}$ is administered prior to incision.

Postoperative analgesia regimen

\section{Intervention group: erector spinae plane}

Patients in the intervention group will receive continuous ESP analgesia with an infusion of $5 \mathrm{ml} / \mathrm{h}$ bupivacaine $0.125 \%$ and a nurse administered bolus of $10 \mathrm{ml}$ bupivacaine $0.125 \%$ every 3 hours. The nurse will get an automated order to give the bolus at regular 3-hour intervals. They also receive a patientcontrolled intravenous analgesia (PCIA) pump with piritramide and droperidol. Settings of the PCIA pump will be according to local institutional protocols. When ESP does not provide a NRS $<4$, the patient will be titrated piritramide intravenous until NRS $<4$.

\section{Control group: thoracic epidural analgesia}

Patients in the control group will receive TEA through a continuous epidural analgesia (CEA) pump with either bupivacaine $0.125 \%$ + sufentanil $1 \mu \mathrm{g} / \mathrm{ml}(8-12 \mathrm{ml} / \mathrm{h}$ with the possibility of a bolus of $4 \mathrm{ml}$ every hour) or ropivacaine $0.2 \%$ + sufentanil $0.5 \mu \mathrm{g} / \mathrm{ml}$ (rate of $5 \mathrm{ml} / \mathrm{h}$ with a $2 \mathrm{ml}$ bolus on demand with a lockout of 20 minutes) according to local institutional protocols. When CEA does not provide a NRS $<4$, an extra bolus of $5 \mathrm{ml}$ of bupivacaine $0.125 \%$ + sufentanil $1 \mu \mathrm{g} / \mathrm{ml}$ or ropivacaine $0.2 \%+$ sufentanil 0.5 $\mu \mathrm{g} / \mathrm{ml}$ will be given.

Discontinuation of ESP or CEA is planned on the morning of day 2, after APS assessment, unless sideeffects or safety issues mandate discontinuation or removal earlier. Patients will receive a daily visit by the acute pain service (APS) team, who will convert the patients to oral analgesics (paracetamol, NSAID and oxycodone) after removal of ESP or CEA. 
All patients will receive the standard pain treatment with paracetamol and NSAIDs following the in house protocol. The attending anesthesiologist will decide if IV titration of piritramide at the end of the surgery is necessary for smooth transition to the post anesthesia care unit. In the PACU ward, intravenous piritramide will be titrated until NRS $\leq 4$. If this is insufficient, intravenous clonidine $1 \mathrm{mcg} / \mathrm{kg}$ will be added.

The APS team will visit the patient daily and adjust the analgesic regimen as described above to manage inadequate analgesia. They will systematically screen for side effects, failure of analgesic technique, need for rescue medication and need for specialist intervention.

\section{Summary of known and potential risks}

In a prospective audit report from the UK, the incidence of permanent injury from neuraxial anesthesia was 4.2 per 100,000. (20) Complications such as total spinal, subdural injection, nerve injury, spinal epidural hematoma an infection are described. (21) The incidence of spinal-epidural hematoma was $1: 18,000$ in a retrospective study in 2004. (22)

For the erector spinae plane block, there is significantly less literature on complications. The most important risk of this block is that of local anesthetic systemic toxicity (LAST), as large volumes of local anesthetic are injected. In a case series published by Tulgar et al of 182 patients undergoing ESP block, complications were reported by $0.22 \%(n=4)$ patients. (23) They were all minor reactions, for example hypotension, and specifically no cardiac events. (24)

In our current expertise, which follows the available literature, we perceive that ESP block is safe compared to the standard treatment, epidural analgesia. It is conducted in an environment with extensive monitoring of vital parameters and direct presence of experts in the field able to provide immediate support if required. The dosing with catheters in the postoperative phase resembles treatment, which is current standard practice in peripheral nerve blocks and postoperative epidural analgesia.

\section{Criteria for discontinuing or modifying allocated interventions $\{11 \mathrm{~b}\}$}

We will exclude patients to participate in the study in case of failure of primary insertion of the ESP or TEA catheter. This will be documented as technical failure. (Table 1) Secondary failures, such as catheter dislodgement, migration or leakage leading to premature removal of the catheter will be documented. Need for rescue medication and need for specialist intervention will also be reported. When the surgical technique changes intra operatively, e.g. conversion to open procedure, the patient will be replaced and will not be used in the intention to treat analysis.

Table 1: definitions of technical complications

\section{Strategies to improve adherence to interventions $\{11 \mathrm{c}\}$}


The APS team will visit the patient daily and adjust the analgesic regimen as described above to manage inadequate analgesia. They will systematically screen for side effects, failure of analgesic technique, need for rescue medication and need for specialist intervention.

\section{Relevant concomitant care permitted or prohibited during the trial $\{11 \mathrm{~d}\}$}

Postoperative pain strategy is outlined in the study protocol. Concomitted care of any other kind is permitted during the trial, if necessary.

\section{Provisions for post-trial care $\{30\}$}

The sponsor/investigator has liability insurance, which is in accordance with article 7 of the WMO. This insurance provides cover for damage to research subjects through injury or death caused by the study.

\section{Outcomes $\{12\}$}

\section{Primary endpoint}

The primary outcome in this study is the Quality of Recovery-15 (QoR-15) score. This score is a compound measure of overall postoperative patient recovery. The score comprises a 15-item questionnaire that provides an evaluation across five dimensions: patient support, comfort, emotions, physical independence and pain. The questionnaire has been validated extensively and a minimal clinically important difference has been defined. (25) We will measure the QoR-15 score before surgery as a baseline, and on postoperative day (POD) 1 and 2 as these questions relate to the 24-hour period covering the day of the surgery and the first postoperative day respectively.

Secondary endpoints

Secondary endpoints are Numerical Rating Scales (NRS) score preoperatively and on POD 0, 1 and 2 (assessed at rest and when moving (coughing) in the morning and in the evening), length of hospital stay (LOS), failure of analgesic technique (defined as catheter failure, need for specialist intervention and/ or need for rescue medication, see table 1), the postoperative morphine-equivalent consumption on POD 0, 1 and 2 , itching, nausea and vomiting, the total operative time (recorded as total time spent in the operating room, anesthetic time, surgical time), complications related to surgery (e.g., bleeding, surgical site infection, conversion to open procedure), perioperative hypotension despite fluid boluses or vasopressor bolus (defined by use of continuous infusion of vasopressors) complications related to the study intervention (e.g., epidural hematoma and abscess or local anesthetic toxicity), duration of bladder catheterization, time of first assisted mobilization ( $>20$ meters and to sitting in a chair).

Other study_parameters 
Upon inclusion we will collect gender, age, weight, height, American Society of Anesthesiologists (ASA) risk score, type of surgery, opioids in home medication

\section{Participant timeline $\{13\}$}

Patients will be asked to enroll in the study during the preoperative anesthesiology consultation. They will be randomized in an ESP group and a TEA group. A baseline assessment of the QoR-15 upon inclusion will be done. Patients will undergo placement of TEA of ESP catheter just prior to surgery on the preoperative ward in the pre-anesthesia room. After surgery, on the recovery ward or ICU, the NRS score will be taken by the recovery nurse or ICU nurse. Pain medication will be given, according to this protocol. Patients will receive a daily visit by the acute pain service (APS) team until they can be converted to oral analgesics: paracetamol, NSAID and oxycodon. Patients will be followed until POD2 or until (earlier) discharge from the hospital. A flowchart of the study design is presented in figure 1.

Figure 1: schedule of enrolment, interventions, and assessments.

Figure 2: Flow chart of the study design.

GA: General Anesthesia. VATS: Video-assisted Thoracoscopic Surgery. BMI: Body Mass Index. ASA: American Society of Anesthesiologists. ESP: Continuous Erector Spinae Plane Analgesia. TEA: Thoracic Epidural Analgesia. QoR15: Quality of Recovery 15 Score

\section{Sample size $\{14\}$}

Based on the study published by Myles, we assumed the standard deviation of the QoR15 to be 18 for video-assisted thoracic surgery (intermediate extent of surgery). (25) From the same study, a noninferiority limit of 13 was assumed as Myles et al. determined that this value represents 'minimal change' for the QoR15. Using a one sided alpha of 0.05 , a standard deviation of 18 and a power of $95 \%, 42$ patients are necessary in each group. With a dropout rate of $7 \%$, the total number of patients to randomize is 90 . The two hospitals have a joint recruitment pool of 180 VATS per year and expect $60 \%$ of the patients to be both eligible and willing to participate. We aim to complete inclusion within $a+/-12$ month period. A total of 90 patients are being randomly allocated to ESP (study group) or TEA (control group).

\section{Recruitment $\{15\}$}

Patients will be asked to participate in the study during the preoperative anesthesiology consultation.

\section{Assignment of interventions: allocation}

\section{Sequence generation $\{16 \mathrm{a}\}$}


In this open label study, patients will be randomly allocated in a 1:1 ratio to receive either an ESP block (study group) or a TEA block (control group) by using a predetermined computer-generated randomized schedule. Only the investigators will have access to this list. Permutated block randomization with varying permuted block sizes will be used to divide the lobectomy and wedge resections in homogenous strata to manage for any surgical variation. Separate randomization lists will be used for the two hospitals, to reduce bias. This blocked randomization list will be created with Sealed EnvelopeTM software.

\section{Concealment mechanism \{16b\}}

Participants will be randomised using Research Manager, My Data Manager which is an online, central randomisation service within the program Research Manager. Allocation concealment will be ensured, as the service will not release the randomisation code until the patient has been recruited into the trial, which takes place after all baseline measurements have been completed.

\section{Implementation $\{16 c\}$}

The person who will generate the allocation sequence is not involved in the study. The attending anesthesiologist on the preoperative anesthesiology clinic will enroll participants. The researchers will assign participants to interventions, according to the randomization.

\section{Assignment of interventions: Blinding}

\section{Who will be blinded $\{17 \mathrm{a}\}$}

Blinding of the trial participants and study personnel engaged in patient care to group allocation is not possible due to the different clinical characteristics and invasive nature of the two study interventions. Statistical analysis will be performed by a third-party who is not otherwise involved in the conduct of the study; they will be fully blinded to the hypothesis of the study and to the group allocation.

\section{Procedure for unblinding if needed $\{17 \mathrm{~b}\}$}

\section{$\mathrm{N} / \mathrm{A}$}

\section{Data collection and management}

\section{Plans for assessment and collection of outcomes $\{18 \mathrm{a}\}$}

Patients' demographical data will be collected upon inclusion assessment during the preoperative anesthesiology consultation. The attending anesthesiologist will collect data with regard to the anesthesia and surgical procedure. Nurses will collect the data at the Post Anesthesia Care Unit (PACU). The APS team will collect QoR15 scores, opioid consumption, NRS scores and adverse events. The amount and frequency of the opioid usage will be extracted out of the PCA pump. NRS scores and additional administration of analgesia will be extracted out of the medical chart of the patient as 
collected by the nurses at the ward. Complications will be assessed on POD 2 or earlier at discharge. Some of the data will be registered on paper. After termination of the trial, the data will be directly registered in the software program My Research Manager.

\section{Plans to promote participant retention and complete follow-up $\{18 \mathrm{~b}\}$}

To reduce non-retention, the APS team will stimulate the participants to complete the questionnaires. Also, most data except for the questionnaires is registered on a regular basis for all patients who have epidural analgesia, therefore this limits registration burden and prevents missing data.

Non-adherence to protocol is described in the lower section on page 8 'Criteria for discontinuing or modifying allocated interventions $\{11 \mathrm{~b}\}$ '. All non-adherence will be documented and reported.

\section{Data management $\{19\}$}

The Monitoring team of the Catharina Hospital Eindhoven will provide the monitoring and quality assurance of this study. Data entry, coding and storage will be done according to GCP-standards. Publication of data will be done anonymously.

\section{Confidentiality $\{27\}$}

The data of each patient will be noted on an individual case report form. Data will be coded using a numerical code, the key to this code is only available to the research team and is stored in the investigator site file in accordance with the Dutch law 'Algemene Vordering Gegevensbescherming (AVG; Personal Data Protection Act) and GCP. All patient data will be handled confidentially and anonymously. Data will then be inserted into a database (GCP validated), and a second investigator will control correctness of entries. All data, including case report forms and consent forms, will be stored for fifteen years after completion of the study. Data, both anonymous or not, will always be stored securely, in a locked cabinet (hard copy) or on password secured computers

\section{Plans for collection, laboratory evaluation and storage of biological specimens for genetic or molecular analysis in this trial/future use $\{33\}$}

N/A, biological specimens are not used in this study.

\section{Statistical methods}

\section{Statistical methods for primary and secondary outcomes $\{20 a\}$}

All statistical analyses will be done in consultation with a senior statistician. The primary analysis will be an intention to treat analysis. Secondary analyses will include a per protocol analysis to identify any protocol deviations or other safety signals. Continuous data will be presented as mean and standard deviation or median and interquartile range. Normality of distributions for continuous variables will be assessed with skewness and kurtosis measures. Continuous data will be assessed using a Student's t- 
test if they are normally distributed or a Mann Whitney $U$ test if otherwise. Dichotomous data will be presented in percentages. Categorical data will be analyzed using a Chi-square test or Fisher exact test.

\section{Primary study_parameter(s).}

We will compare the (mean) score of QoR15 of each day separately between the standard and the intervention group using the Student's t-test. Repeated-measures analysis will be used to assess changes over time in the study parameters.

Secondary study_parameter(s).

Continuous data (postoperative morphine-equivalent consumption per day, postoperative NRS score on POD 1 and 2; length of hospital stay, requirement of rescue medication, failure of anesthetic technique, total operative time, complications related to surgery or pain treatment, duration of bladder catheterization, first assisted mobilization to chair and $>20$ meters, 30 day postsurgical evaluation) will be assessed using a Student's t-test if they are normally distributed or a Mann Whitney $U$ test if otherwise. Categorical data (e.g., gender) will be analyzed using a Chi-square test or Fisher exact test. The mean difference between the two groups will be presented together with the $95 \%$ confidence interval.

\section{Interim analyses $\{21 \mathrm{~b}\}$}

An interim-analysis will be performed after randomization of $50 \%$ of the patients. This analysis will be performed by the Education and Research team of our hospital, more specifically a professional in statistical analysis, who is not engaged in the anesthesiology practice or with our (study) patients. Criteria for terminating the study prematurely are indicators of insufficient patient comfort and are defined as mean NRS $>6$ of all patients during more than 2 consecutive measurements in the study group in the first 24 hours or mean QoR15 $<83$ in the study group on POD2.

\section{Methods for additional analyses (e.g. subgroup analyses) $\{20 \mathrm{~b}\}$}

$\mathrm{N} / \mathrm{A}$

\section{Methods in analysis to handle protocol non-adherence and any statistical methods to handle missing data $\{20 \mathrm{c}\}$}

Primary analysis will be an intention to treat analysis. Secondary analysis will include a per protocol analysis. Within the $7 \%$ drop out safety buffer, there will be no replacement of study patients. A drop out is defined as a patient not completing the full duration of the study. When the surgical technique changes intra operatively, e.g. conversion to open procedure, the patient will be replaced and will not be used in the intention to treat analysis.

There will be no follow-up of patients withdrawn from the treatment if they withdrawal before at least the QoR15 score on POD1 is registered. In that case, the patient will be replaced after we exceeded the 7\% 
withdrawal safety buffer. Data of the dropouts with a QoR15 score on POD1 will be used in the intention to treat analysis.

\section{Plans to give access to the full protocol, participant level-data and statistical code $\{31 \mathrm{c}\}$}

The datasets generated during and/or analyzed during the current study will be made available from the corresponding author on reasonable request and will only be accessible to personnel involved in the trial.

\section{Oversight and monitoring}

\section{Composition of the coordinating centre and trial steering committee $\{5 \mathrm{~d}\}$}

The Catharina Hospital is the coordinating centre. Arthur Bouwman is lead investigator. Renee van den Broek is the main researcher at the Catharina Hospital, Seppe Koopman is the main researcher at the Maasstad Hospital. They are responsible for identification, recruitment, data collection and completion of $\mathrm{CRFs}$, along with follow up of study patients and adherence to study protocol. A senior statistician will be consulted for statistical analysis. All protocol contributors will draft and/or revise the manuscript.

\section{Composition of the data monitoring committee, its role and reporting structure $\{21 \mathrm{a}\}$}

The Monitoring team of the Catharina Hospital Eindhoven will provide the monitoring and quality assurance of this study. The monitor for this study is Sylvie Kolfschoten, department of research and education, Catharina Hospital, the Netherlands.

This study has a low risk and there is no need for a DSMB (Data Safety Monitoring Board). The technique used does not pose extra safety risks compared to the standard treatment and is conducted in an environment with extensive monitoring of vital parameters and direct presence of experts in the field able to provide immediate support if required. The dosing with catheters in the postoperative phase resembles treatment, which is current standard practice in peripheral nerve blocks and postoperative epidural analgesia.

\section{Adverse event reporting and harms $\{22\}$}

In accordance to section 10 , subsection 4 , of the WMO, the sponsor (in this investigator-initiated study, the investigator) will suspend the study if there is sufficient ground that continuation of the study will jeopardize subject health or safety. The investigator will notify the accredited MREC (Medical Research Ethics Committee) without undue delay of a temporary halt including the reason for such an action. The study will be suspended pending a further positive decision by the accredited MREC. The investigator will take care that all subjects are kept informed.

Adverse events are defined as any undesirable experience occurring to a subject during the study, whether or not considered related to the trial procedure. All adverse events reported spontaneously by the subject or observed by the investigator or his staff will be recorded. 
A serious adverse event is any untoward medical occurrence or effect that

- $\quad$ results in death;

- $\quad$ is life threatening (at the time of the event);

- $\quad$ requires hospitalization or prolongation of existing inpatients' hospitalization;

- $\quad$ results in persistent or significant disability or incapacity;

- $\quad$ is a congenital anomaly or birth defect; or

- $\quad$ any other important medical event that did not result in any of the outcomes listed above due to medical or surgical intervention but could have been based upon appropriate judgment by the investigator.

An elective hospital admission will not be considered as a serious adverse event.

The investigator will report all SAEs to the sponsor without undue delay after obtaining knowledge of the events, except for the following SAEs: surgical or other complications unrelated to the anesthetic procedure.

The sponsor will report the SAEs through the web portal ToetsingOnline to the accredited MREC that approved the protocol, within 7 days of first knowledge for SAEs that result in death or are life threatening followed by a period of maximum of 8 days to complete the initial preliminary report. All other SAEs will be reported within a period of maximum 15 days after the sponsor has first knowledge of the serious adverse events.

\section{Follow-up of adverse events}

All AEs will be followed until they have abated, or until a stable situation has been reached. Depending on the event, follow up may require additional tests or medical procedures as indicated, and/or referral to the general physician or a medical specialist. SAEs need to be reported till end of study within the Netherlands, as defined in the protocol

\section{Frequency and plans for auditing trial conduct $\{23\}$}

The Monitoring team of the Catharina Hospital Eindhoven will provide the monitoring and quality assurance of this study. The monitor will perform an initiation visit and will audit the overall quality and completeness of the data, examine source documents and confirm that the clinical centers have complied with the requirements of the protocol. The monitor will verify that all adverse events were documented in the correct format, and are consistent with protocol definition.

Plans for communicating important protocol amendments to relevant parties (e.g. trial participants, ethical committees) $\{25\}$ 
Amendments are changes made to the research protocol after a favorable opinion by the accredited ethics committee. All amendments will be notified to the ethics committee.

\section{Dissemination plans $\{31$ a $\}$}

The trial's results will be submitted to a peer-reviewed journal regardless of the outcome.

\section{Discussion}

Although the ESP block is gaining popularity as analgesia in thoracic surgery, there has been limited study of its clinical efficacy compared with the current gold standard of thoracic epidural analgesia (TEA). However, these preliminary results are promising. $(2-4,26-28)$ This prompted us to speculate that ESP block may have a similar analgesic profile to TEA, and in turn to conduct a trial to compare these two techniques and further validate the theory from a clinical aspect.

The expected results will provide clinical evidence to verify the analgesic mechanism of ESP and promote its application in minimally invasive thoracic surgery. Furthermore, if the efficacy of ESP is equal to that of TEA, ESP could replace TEA in minimally-invasive thoracic surgery because it is relatively safe and convenient to perform, while TEA carries risks of failure, dural puncture and epidural abscess or hematoma. $(14,20,21,29)$ Furthermore, unlike TEA, ESP does not require routine urinary catheterization to manage urinary retention and thus facilitates the early mobilization of the patient after surgery.

The proposed trial is a prospective, randomized trial with rigorous methodology to avoid potential risk of bias. Since the nature of the intervention makes it impossible for the participant, the investigator performing the block, and the outcome assessor to be blinded, only the statistician will be blinded to the allocation to keep the data analysis unbiased.

In contrast to epidural analgesia, ESP analgesia can only induce a unilateral sensory block of thoracic dermatomes. Nonetheless, this unilateral thoracic block is expected to achieve adequate control of chest pain after minimally invasive thoracic surgery, especially when combined with patient-controlled intravenous opioid analgesia as an escape for breakthrough discomfort. (3-5) If ESP analgesia in fact achieves adequate pain control, the avoidance of epidural-related side-effects likely facilitates fast recovery after minimally invasive thoracic surgery, which is the primary interest of this study. Since postoperative recovery is multifactorial, a composite endpoint was considered to be most appropriate and therefore the QoR-15 questionnaire was chosen as primary outcome. The QoR-15 consists of 15 questions that are divided in separate sections that aim to evaluate the presence and extent of pain, symptoms, comfort, emotional well-being, physical independence, and satisfaction with treatment. (30) As the hypothesized advantages of ESP analgesia are expected to impact several of these sections, the composite QoR-15 score is considered to be a good parameter for postoperative recovery.

Our evaluation through the QoR15 score matches the call for more patient-centered outcomes in ESP block studies. $(15,31)$ The reduction in pain scores itself may not equate to an improvement in patients 
experience. Factors other than only analgesia, such as general well-being, nausea, itching, being able to get out of bed, appetite and the need for a bladder catheter also matter in the patients' perspective on good recovery, and contribute to enhanced recovery and earlier hospital discharge.

If our trial yields positive results, there is potential that ESP could be recommended as an alternative block for postoperative analgesia in minimally invasive thoracic surgery, to circumvent the need for TEA.

\section{Trial Status}

This document is based on version 5 of the original protocol, approved by the MET-U (ethics committee) on $26^{\text {th }}$ February 2020 , registration number R18.022. We anticipate randomizing the first patient in July 2020 and plan to complete the study in December 2021.

\section{Abbreviations}

ESP: Erector spinae plane

TEA: Thoracic epidural analgesia

VATS: Video-assisted thoracoscopic surgery

QoR15: Quality of recovery

NRS: Numeric Rating Scale

LOS: Length of stay

POD: postoperative day

ASA: American society of anesthesiology

NSAID: non-steroidal anti-inflammatory drug

INR: international normalized ratio

NIBP: non-invasibe blood pressure

ECG: electrocardiogram

BMI: body mass index

CEA: continuous epidural analgesia

APS: acute pain service

LAST: local anesthetic systemic toxicity 
PACU: post anaesthesia care unit

PCIA: patient controlled intravenous analgesia

AE: adverse event

SAE: serious adverse event

GCP: good clinical practice

DSMB: Data Safety Monitoring Board

MREC: Medical Research Ethics Committee

\section{Declarations}

\section{Acknowledgements}

The authors thank Saskia Houterman for her valuable support in drafting of the statistical analysis plan

\section{Authors' contributions \{31b\}}

RB is the corresponding author, she drafted the manuscript and is the main researcher of the study at Catharina hospital. $A B$ is the lead investigator, helped design the study, contributed to and revised the manuscript. BV conceived the project, obtained funding for the project, designed the study, contributed to and revised the manuscript. JK revised the manuscript and is the main researcher in the Maasstad Hospital. JP revised the manuscript and will conduct the study in the Maasstad Hospital. KC helped design the study and revised the manuscript. NV revised the manuscript. All authors read and approved the final manuscript

\section{Funding $\{4\}$}

This work was supported by the Stichting Onderzoeksfonds Catharina Ziekenhuis (Project 2018-7). The funding body had no role in the design of the study, collection, analysis, interpretation of data or in writing the manuscript

\section{Availability of data and materials $\{29\}$}

The lead investigator $A B$ and both $\mathrm{RB}$ and $\mathrm{BV}$ will have access to the final trial dataset. Other contributors of this protocol are allowed access to the final trial dataset upon reasonable request.

\section{Ethics approval and consent to participate $\{24\}$}

The study has approval from the MEC-U, the local medical research ethics committee. The study will be performed in accordance with the Declaration of Helsinki (Fortaleza, Brazil, October 2013) and in 
accordance with the Medical Research Involving Human Subjects Act (WMO) and Good Clinical Practice guidelines. Informed consent will be obtained from all study participants.

\section{Consent for publication $\{32\}$}

This document does not contain personal data and consent for publication is not applicable. We are willing to provide a model consent form on request.

\section{Competing interests $\{28\}$}

The principal investigators have no financial or other competing interests for the overall trial

\section{Authors' information (optional)}

$\mathrm{N} / \mathrm{A}$

\section{References}

1. Scimia P, Basso Ricci E, Droghetti A, Fusco P. The Ultrasound-Guided Continuous Erector Spinae Plane Block for Postoperative Analgesia in Video-Assisted Thoracoscopic Lobectomy. Reg Anesth Pain Med. August 2017;42(4):537.

2. Adhikary SD, Pruett A, Forero M, Thiruvenkatarajan V. Erector spinae plane block as an alternative to epidural analgesia for post-operative analgesia following video-assisted thoracoscopic surgery: A case study and a literature review on the spread of local anaesthetic in the erector spinae plane. Indian J Anaesth. January 2018;62(1):75-8.

3. Kim Eun Soo. A Randomized Controlled Trial Comparing Continuous Erector Spinae Plane Block with Thoracic Epidural Analgesia for Postoperative Pain Management in Video-assisted Thoracic Surgery [Internet]. [Cited on March 19 2020]. Available on:

http://www.asaabstracts.com/strands/asaabstracts/abstract.htm? year=2019\&index=18\&absnum=1958

4. Yao Y, Fu S, Dai S, Yun J, Zeng M, Li H, e.a. Impact of ultrasound-guided erector spinae plane block on postoperative quality of recovery in video-assisted thoracic surgery: A prospective, randomized, controlled trial. J Clin Anesth. March 2020;63:109783.

5. Ciftci B, Ekinci M, Celik EC, Tukac IC, Bayrak Y, Atalay YO. Efficacy of an Ultrasound-Guided Erector Spinae Plane Block for Postoperative Analgesia Management After Video-Assisted Thoracic Surgery: A Prospective Randomized Study. J Cardiothorac Vasc Anesth. February 2020;34(2):444-9.

6. De Cosmo G, Aceto P, Gualtieri E, Congedo E. Analgesia in thoracic surgery: review. Minerva Anestesiol. June 2009;75(6):393-400.

7. Mungroop TH, Veelo DP, Busch OR, Dieren S van, Gulik TM van, Karsten TM, e.a. Continuous wound infiltration versus epidural analgesia after hepato-pancreato-biliary surgery (POP-UP): a randomised controlled, open-label, non-inferiority trial. Lancet Gastroenterol Hepatol. October 2016;1(2):105-13. 
8. Horlocker T. Regional anesthesia in the anticoagulated patient: Defining the risks (the second ASRA Consensus Conference on Neuraxial Anesthesia and Anticoagulation). Reg Anesth Pain Med. May 2003;28(3):172-97.

9. Wedel D, Horlocker T. Regional Anesthesia in the Febrile or Infected Patient. Reg Anesth Pain Med. July 2006;31(4):324-33.

10. Gottschalk A, Cohen SP, Yang S, Ochroch EA. Preventing and treating pain after thoracic surgery. Anesthesiology. March 2006;104(3):594-600.

11. Forero M, Adhikary SD, Lopez H, Tsui C, Chin KJ. The Erector Spinae Plane Block: A Novel Analgesic Technique in Thoracic Neuropathic Pain. Reg Anesth Pain Med. September 2016;41(5):621-7.

12. Kot P, Rodriguez P, Granell M, Cano B, Rovira L, Morales J, e.a. The erector spinae plane block: a narrative review. Korean J Anesthesiol. June 2019;72(3):209-20.

13. Pourkashanian A, Narayanan M, Venkataraju A. The Erector Spinae Plane Block: A Review of Current Evidence. ATOTW. December 2019;11.

14. Taketa Y, Irisawa Y, Fujitani T. Comparison of ultrasound-guided erector spinae plane block and thoracic paravertebral block for postoperative analgesia after video-assisted thoracic surgery: a randomized controlled non-inferiority clinical trial. Reg Anesth Pain Med. January 2020;45(1):10-5.

15. Myles PS. Measuring quality of recovery in perioperative clinical trials. Curr Opin Anaesthesiol. August 2018;31(4):396-401.

16. Swanson SJ, Herndon JE, D’Amico TA, Demmy TL, McKenna RJ, Green MR, e.a. Video-assisted thoracic surgery lobectomy: report of CALGB 39802--a prospective, multi-institution feasibility study. J Clin Oncol Off J Am Soc Clin Oncol. November 2007;25(31):4993-7.

17. Shigemura N, Akashi A, Nakagiri T, Ohta M, Matsuda H. Complete versus assisted thoracoscopic approach: a prospective randomized trial comparing a variety of video-assisted thoracoscopic lobectomy techniques. Surg Endosc. October 2004;18(10):1492-7.

18. Byrne K, Smith C. Human volunteer study examining the sensory changes of the thorax after an erector spinae plane block. Reg Anesth Pain Med. February 2020; 45(10):761-2. doi: 10.1136/rapm2019-101019. Epub 2020 Feb

19. Schwartzmann A, Peng P, Maciel MA, Alcarraz P, Gonzalez X, Forero M. A magnetic resonance imaging study of local anesthetic spread in patients receiving an erector spinae plane block. Can J Anesth Can Anesth 2020;67:942-8 https://doi.org/10.1007/s12630-020-01613-8

20. Cook TM, Counsell D, Wildsmith J a. W. Major complications of central neuraxial block: report on the Third National Audit Project of the Royal College of Anaesthetistst. Br J Anaesth. February 2009;102(2):179-90.

21. Ituk, Wong. Overview of neuraxial anesthesia. In: Maniker, Crowley. Up To Date [Database on the internet]. Waltham (MA): UpToDate; 2020 [Cited on March 11 2020]. Available from: http://www.uptodate.com

22. Moen V, Dahlgren N, Irestedt L. Severe neurological complications after central neuraxial blockades in Sweden 1990-1999. Anesthesiology. October 2004;101(4):950-9. 
23. Tulgar S, Selvi O, Senturk O, Serifsoy TE, Thomas DT. Ultrasound-guided Erector Spinae Plane Block: Indications, Complications, and Effects on Acute and Chronic Pain Based on a Single-center Experience. Cureus [Internet]. [Cited on March 30 2020];11(1). Available from: https://www.ncbi.nlm.nih.gov/pmc/articles/PMC6402723/

24. Fang $B$, Wang Z, Huang $X$. Ultrasound-guided preoperative single-dose erector spinae plane block provides comparable analgesia to thoracic paravertebral block following thoracotomy: a single center randomized controlled double-blind study. Ann Transl Med [Internet]. April 2019 [Cited on March 11 2020];7(8). Available from: https://www.ncbi.nlm.nih.gov/pmc/articles/PMC6526263/

25. Myles PS, Myles DB, Galagher W, Chew C, MacDonald N, Dennis A. Minimal Clinically Important Difference for Three Quality of Recovery Scales. Anesthesiology. July 2016;125(1):39-45.

26. Forero M, Rajarathinam M, Adhikary S, Chin KJ. Erector spinae plane (ESP) block in the management of post thoracotomy pain syndrome: A case series. Scand J Pain. 2017;17:325-9.

27. Luis-Navarro JC, Seda-Guzmán M, Luis-Moreno C, López-Romero JL. The erector spinae plane block in 4 cases of video-assisted thoracic surgery. Rev Esp Anestesiol Reanim. April 2018;65(4):204-8.

28. Nath S, Bhoi D, Mohan VK, Talawar P. USG-guided continuous erector spinae block as a primary mode of perioperative analgesia in open posterolateral thoracotomy: A report of two cases. Saudi J Anaesth. September 2018;12(3):471-4.

29. Chin KJ, McDonnell JG, Carvalho B, Sharkey A, Pawa A, Gadsden J. Essentials of Our Current Understanding: Abdominal Wall Blocks. Reg Anesth Pain Med. March 2017;42(2):133-83.

30. Kleif J, Waage J, Christensen KB, Gögenur I. Systematic review of the QoR-15 score, a patientreported outcome measure measuring quality of recovery after surgery and anaesthesia. $\mathrm{Br} \mathrm{J}$ Anaesth. January 2018;120(1):28-36.

31. Grocott HP. Erector Spinae Plane Block for Postoperative Analgesia: A Magic Bullet Too Good to Be True, or Is It Just Too Soon to Tell? Anesth Analg. 2020 Jan;130(1):e29. doi:

10.1213/ANE.0000000000004428

\section{Table}

Table 1: Definitions of technical complications

\begin{tabular}{|l|l|}
\hline Technical failure & Failure to place a catheter \\
\hline Catheter failure & $\begin{array}{l}\text { Catheter dislodgement, migration or leakage, leading to premature } \\
\text { removal of the catheter. }\end{array}$ \\
\hline $\begin{array}{l}\text { Need for rescue } \\
\text { medication }\end{array}$ & $\begin{array}{l}\text { Patients remain painful (NRS > 4) after maximum adjustments } \\
\text { possible }\end{array}$ \\
\hline $\begin{array}{l}\text { Need for } \\
\text { specialist } \\
\text { intervention }\end{array}$ & $\begin{array}{l}\text { Suboptimal functioning of CEA/ESP on ward leading to supervision by } \\
\text { an anesthesiologist and/or minor adjustments }\end{array}$ \\
\hline
\end{tabular}


Figures

\begin{tabular}{|c|c|c|c|c|c|c|c|}
\hline & Enrolment & Allocation & & & & & \\
\hline TIMEPOINT & $\begin{array}{l}\text { Preop } \\
\text { anesthesiol } \\
\text { ogy consult }\end{array}$ & POD-1 & $\begin{array}{l}P O D \\
0\end{array}$ & $\begin{array}{l}P O D \\
1\end{array}$ & $\begin{array}{l}P O D \\
1\end{array}$ & $\begin{array}{l}P O D \\
2\end{array}$ & $\begin{array}{l}P O D \\
2\end{array}$ \\
\hline \multicolumn{8}{|l|}{ ENROLMENT: } \\
\hline Eligibility screen & $\mathrm{x}$ & & & & & & \\
\hline Informed consent & $\mathrm{x}$ & & & & & & \\
\hline Allocation & & $\mathrm{x}$ & & & & & \\
\hline \multicolumn{8}{|l|}{ INTERVENTIONS: } \\
\hline \multicolumn{8}{|l|}{ ESP } \\
\hline \multicolumn{8}{|l|}{ TEA } \\
\hline \multicolumn{8}{|l|}{ ASSESSMENTS: } \\
\hline \multirow{2}{*}{$\begin{array}{l}\text { QoR15 } \\
\text { Other outcome } \\
\text { variables, see fig } 2\end{array}$} & $\mathrm{x}$ & & & $\mathrm{x}$ & $\mathrm{x}$ & $\mathrm{x}$ & $\mathrm{x}$ \\
\hline & & & & $\mathrm{x}$ & $\mathrm{x}$ & $\mathrm{x}$ & $\mathrm{x}$ \\
\hline
\end{tabular}

Figure 1

Schedule of enrolment, interventions, and assessments. 


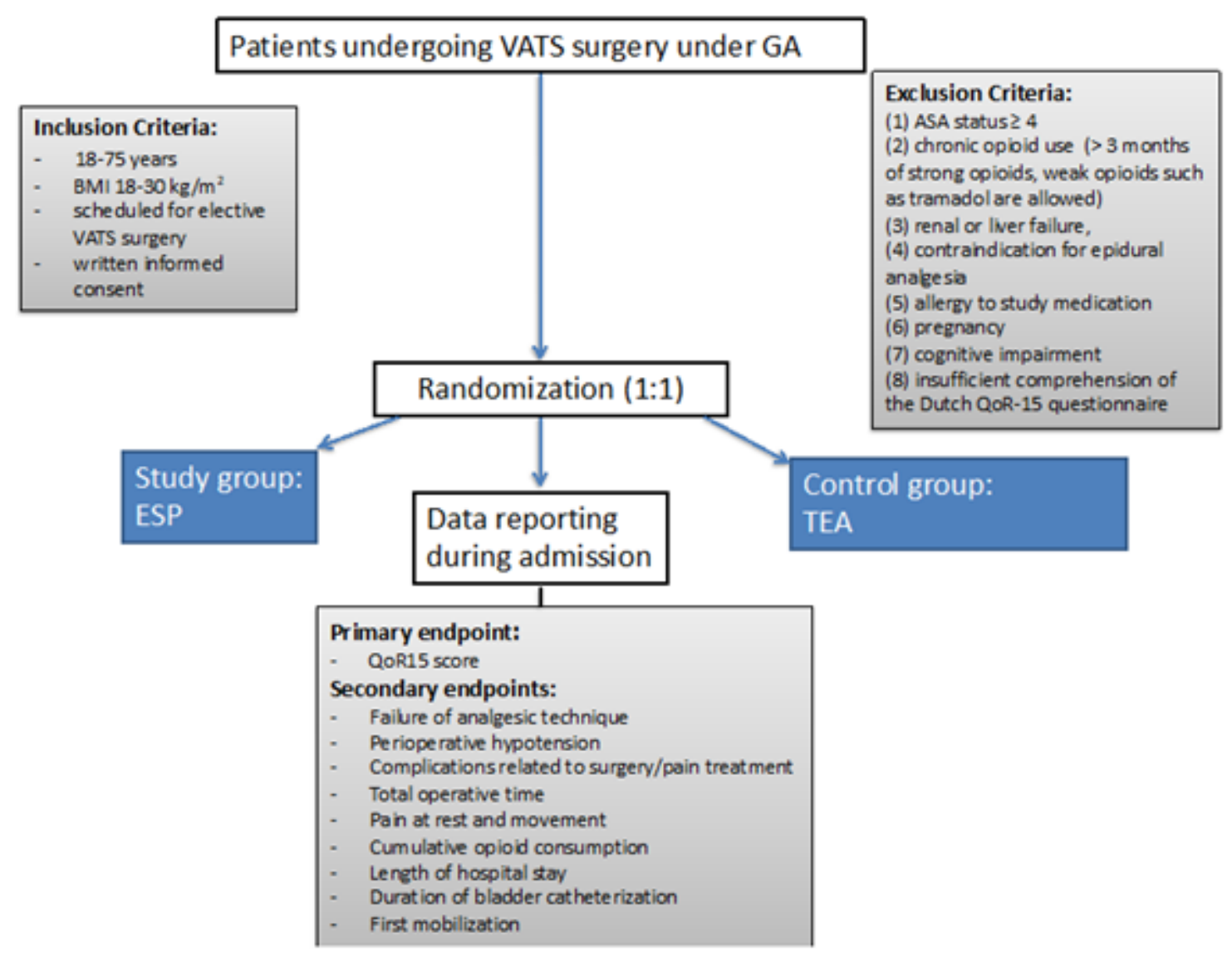

Figure 2

Flow chart of the study design. 